\title{
On Null Hypotheses and Heteroskedasticity
}

\author{
Jeffery Sauer · Taylor Oshan · Sergio Rey • \\ Levi John Wolf
}

\begin{abstract}
Bivand and Wong (2018), a recent review on spatial statistical software, noted important differences in the results of the local Moran's $I_{i}$ statistic depending on the method of inference. That review speculated the differences may be due to the presence of local spatial heterogeneity. In this paper we design an experiment to assess the impact of local heterogeneity on hypothesis testing for local statistics. In this experiment, we analyze the relationship between measures of local variance, such as the local spatial heteroskedasticity (LOSH) statistic, and components of the local Moran's $I_{i}$ statistic. We consider this experiment with controlled synthetic heteroskedastic data and with uncontrolled real world data. We show that in both situations the variance components of the local Moran's $I_{i}$ statistic demonstrate a varying correlation with alternative measures of local variance like LOSH. In addition, we resituate the available inferential methods and suggest an alternative explanation for the differences observed in Bivand and Wong 2018. Ultimately, this paper demonstrates that there are important conceptual and computational differences as to what constituents a null hypothesis in local testing frameworks. Therefore, researchers must be aware as to how their choices may shape the observed spatial patterns.
\end{abstract}

Keywords LOSH · local Moran statistic - Spatial clusters - Spatial variance · Spatial data science

J. Sauer

Department of Geography, University of Maryland, College Park, USA

Tel.: +16123941115

E-mail: jcsauer@terpmail.umd.edu

T.M. Oshan

Department of Geography, University of Maryland, College Park, USA

S. Rey

School of Public Policy, University of California Riverside, Riverside, USA

L.J. Wolf

School of Geographical Sciences, University of Bristol, Bristol, UK 


\section{Introduction}

Describing spatial autocorrelation is a fundamental objective of exploratory spatial analysis (Getis, 2008). Several scholars have contributed statistics, inference, and visualization techniques to express the structure of geographical relationships. Often, analysts are interested in local statistics that characterize geographical patterns at a specific site in the geographical pattern. The local Moran's $I_{i}$ statistic is one such highly influential example that has been applied across a range of disciplines to explore patterns of spatial autocorrelation. Originally developed by Anselin (1995), it characterizes areas of a geographical pattern into "hotspots," areas where observations tend to be very similar, and "spatial outliers," where areas are very different from their surroundings. Later extensions have expanded the statistic to consider multivariate relationships (Anselin and Li, 2020; Anselin et al., 2002), and connect the statistics to other measures of association (Lee, 2001).

However useful statistics are, they become more useful when attached to an inferential framework. This enables us to make decisions about how large (or small) a statistic is with respect to a theory about what a typical distribution the statistic should be, under a few assumptions. The original presentation of the local Moran's $I_{i}$ in Anselin (1995) provided analytical expressions for the mean and variance of local $I_{i}$ statistics, but strongly encouraged analysts to use a simulation-driven strategy for inference, sometimes called "conditional permutation." This simulation-driven inference is used in some implementations, but not all (Bivand and Wong, 2018), and is generally omitted because it is computationally-intensive. Therefore, while many initial issues with testing have generally been addressed, such as those for multiple testing (Caldas de Castro and Singer, 2006) and for event variables occurring among unequal populations (Assuncao and Reis, 1999), work continues on additional methods of inference (both approximate in Tiefelsdorf (2002) and exact in Bivand et al. (2009)) that avoid computing simulations.

Something common to all of the inference methods is the use of null hypothesis statistical testing. That is, any of the inferential methods, simulationdriven or analytical, refer to a specific hypothesis about spatial randomness, against which the observed values of local statistics are compared. In the past, specifying a "reasonable" null has been somewhat controversial. For example, in attempting to address issues of testing local statistics when global autocorrelation is present, Ord and Getis (2001) note significant disagreements about reasonable nulls and appropriate comparisons for statistical tests ( $p$. 419). Slightly earlier Sokal et al. (1998) investigated issues pertaining to these nulls, specifically focusing on the concept of "conditional" during permutationdriven inference. Regardless, statistical software packages (Bivand et al., 2011; Rey and Anselin, 2007) tend to implement a subset of inference methods from the literature and encourage users to be aware of differences.

More recently, a thorough review of local statistics across software packages by Bivand and Wong (2018) compared various inference methods for local Moran's $I_{i}$, among other local statistics. They found that the methods 


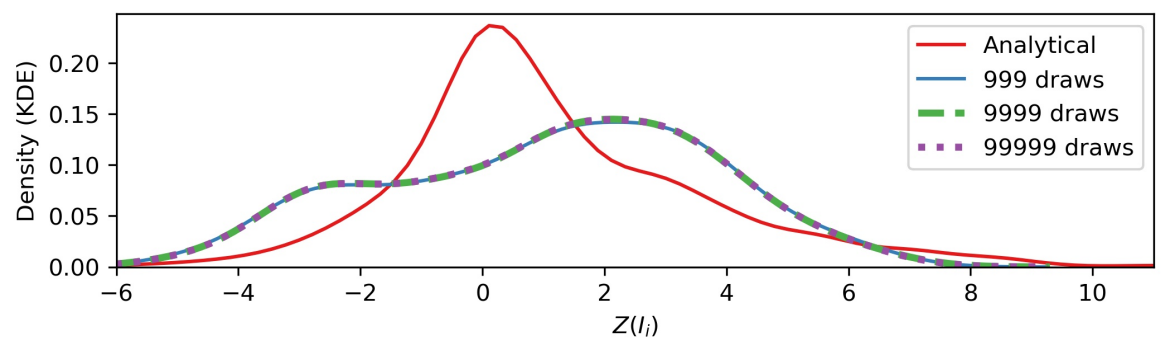

Fig. 1 Recreation of Figure 8 from Bivand and Wong (2018), calculated on Output Area measurements of unemployment. The plot shows the distributions of realizations from a conditional permutation strategy, versus analytical results. As explained in the source, increasing the number of permutations does not lead to a substantive change in the distributions. The similarity between the permutation distributions leads to visual overplotting.

provided different substantive results, with serious differences in the numbers of "significant" local statistics. Thus, concerns about local statistics, nulls, and inference still have not been definitively addressed.

In the end, Bivand and Wong speculated that the cause of these differences may be due to observed local spatial heterogeneity, but they did not demonstrate this definitively. In the present paper, we briefly summarize Bivand and Wong's core findings. Then, we leverage results from Sokal et al. (1998) that provide a better theoretical framework for analyzing local statistical tests. With this framework, we can directly examine what drives the differences observed by Bivand and Wong. For empirical evidence, we estimate the relationship between a measure of local spatial heteroskedasticity Ord and Getis (2012) and analytical (or simulated) moments of the local Moran's $I_{i}$ statistics. We use two reproducible ${ }^{1}$ experimental scenarios. The first scenario represents a common situation in empirical research wherein the underlying data generating process (DGP) for a phenomenon of interest is unknown. The second scenario is a controlled DGP where we purposefully induce spatial heteroskedasticity. We find that it is more likely that differences in null hypotheses, not local spatial heteroskedasticity, drive the differences in inference observed in Bivand and Wong (2018). This means that end users need to be aware of the implications of their null hypotheses when conducting local statistical inference, a finding that reinforces contemporary critique about the epistemology of applied statistics generally (Wasserstein and Lazar, 2016). 


\section{Revisiting Bivand and Wong's observations on local Moran's I}

To start, we reprise the discussion of Bivand and Wong, who deploy measures of spatial autocorrelation on an empirical example concerning the proportion of the population unemployed for Output Areas (OA) in the London Borough of Camden (Bivand and Wong, 2018). Despite consistent agreement throughout the rest of the experiments assessed by Bivand and Wong, the distribution of local Moran's $I_{i}$ z-scores, $Z\left(I_{i}\right)$, were distinctly different when using analytical versus conditional permutation methods of inference. ${ }^{2}$ This difference is perhaps most evident in their comparison of the density plots for $Z\left(I_{i}\right)$ values generated by analytical and permutation-based inference: the distribution for conditional permutation $Z\left(I_{i}\right)$ scores is flatter and wider (Figure 1, reproduced from Figure 8 in Bivand and Wong 2018). These observed differences in inference on local Moran's $I_{i}$ statistics are our key interest. ${ }^{3}$

\subsection{Differences in Analytical and Permutation-based Local Statistics}

A few major differences in the inferential results emerge when comparing analytical and permutation-based local statistics. First, the permutation-based local $z$-scores were much more likely to be statistically significant, compared to the analytically-based local scores. Analytical inference yielded 267 significant $\mathrm{OAs}^{4}$ ( $45.6 \%$ of total OAs), whereas permutation-based inference yielded 429 OAs (57.2\% of total OAs). Second, entirely different OAs attained statistical significance. Third, increasing the number of permutations did not remedy the observed gap between inference methods, nor did adjusting the $p$-values by the false discovery rate.

These differences have serious effects. Applied scientists who are not familiar with the nuances of the underlying methods will not know that their inference method influences their results. Generally, users accept software defaults and it is reasonable to assume that different inference strategies will arrive at similar results. Further, only a subset of inference methods are available in any given software package (Bivand and Wong, 2018), so it may not be easy to check whether inference methods do substantively agree. Most critically, it is not possible to determine which is "correct" outside of strictly controlled settings. Therefore, when Bivand and Wong find inference methods disagree in this manner, it is imperative to understand why.

1 The entirety of this analysis including instructions on data acquisition, code for analysis, and figure creation is maintained via a private Github repository and can be reproduced from a single Jupyter Notebook. The repository will be made public after receiving decision from publication. We provide a copy of the Jupyter Notebook, which includes instructions on data acquisition, in the submission files.

2 Differences are also observed in $z$-scores for local $G_{i}$ statistics, $Z\left(G_{i}\right)$, but here the conditional permutation distribution is much narrower and are driven by differences in how zero-neighbor observations are treated (Bivand and Wong, 2018, pg. 734-735).

${ }^{3}$ We encourage interested readers to seek out additional detail in Bivand and Wong's extensive review.

${ }^{4}$ Here, significance is attained when $\left|Z\left(I_{i}\right)\right|>2$, implying an observed local $I$ is more than two standard errors away from an estimate of the local $I_{i}$ under a null hypothesis of no clustering. 


\subsection{Speculation on Heteroskedasticity and Local Tests}

In concluding their paper, Bivand and Wong speculate that this discrepancy between analytical and permutation-based Local Moran z-score distributions may be due to local spatial heterogeneity. This could be conceptualized as the presence of localized, unequal variance in the data (Ord and Getis, 2012). Put simply, this may happen when some areas of a surface are more "noisy" than others. Local heteroskedasticity may invalidate assumptions about the variance of $I_{i}$ (Anselin, 1995; Ord and Getis, 2012), whose local values vary based on the structure of the spatial weights matrix. Indeed, Bivand and Wong calculate local heteroskedasticity (using $\mathrm{LOSH}, \mathrm{H}_{i}$, from Ord and Getis (2012)) for their empirical example and note the presence of local spatial heteroskedasticity.

However, there are other possibilities. Bivand and Wong's analytical inference derives from Anselin (1995) and Sokal et al. (1998). In addition to providing a computational simplification for Anselin's estimator, Sokal et al. (1998) also re-evaluates the assumptions made by null hypotheses for inference on local Moran's $I_{i}$. Sokal calls the Anselin (1995) form a "total randomization" null, and contrasts it with a "conditional" randomization version that is expressed analytically. This provides a different expression for $Z\left(I_{i}\right)$ that assumes that the values at each site are fixed during randomization at that site. Comparing the two approaches, Sokal "do[es] not find substantial differences" among simulated $I_{i}$, but does note that:

skewness and kurtosis are more pronounced in the total randomization replicates than in those randomized conditionally. Significance tests based on conditional randomization assumptions might thus be more reliable (Sokal et al., 1998, pg. 349)

Bivand and Wong use the "total" randomization null hypothesis from Anselin (1995) when conducting what they call "analytical" inference, and compare this to the conditional permutation results from Rey and Anselin (2007) (which are also simulation-based) which use a different implied null hypothesis. That is, two things are changing in this comparison: the null ("total" versus "conditional") and the method ("analytical" versus "simulationbased"). We demonstrate these various combinations of null hypotheses and inference methods as they have appeared in Figure 2.

\section{Local Statistics}

Therefore, there are at least two possibilities driving the differences observed in Bivand and Wong (2018). For one, local heteroskedasticity in the data may cause the local $Z\left(I_{i}\right)$ statistics to inflate. Alternatively, different assumptions embedded within our null hypotheses will change both the expectation and variance used to compute $Z\left(I_{i}\right)$. Both can be true at the same time, too. 


\begin{tabular}{|c|c|c|}
\hline & $\begin{array}{l}\text { "Total" Randomization Null } \\
\text { all permutations of values are equally } \\
\text { likely at any site }\end{array}$ & $\begin{array}{l}\text { "Conditional" Randomization Null } \\
\text { for each site, the site's value(s) are } \\
\text { fixed and remaining sites are shuffled }\end{array}$ \\
\hline $\begin{array}{l}\text { Analytical inference } \\
\text { closed-form mathematical } \\
\text { "reference" distribution }\end{array}$ & $\begin{array}{l}\text { Originally in Anselin (1995). } \\
\text { Implemented in Rey \& Anselin (2007), } \\
\text { Bivand et al. (2011). } \\
\text { Used in Bivand \& Wong (2018). } \\
\text { Denoted here as } E\left[I_{i}\right] \text { and } \operatorname{Var}\left[I_{i}\right]\end{array}$ & $\begin{array}{l}\text { Originally in Sokal (1998). } \\
\text { Implemented in this paper. } \\
\text { Not used in Bivand \& Wong (2018). } \\
\text { Denoted here as } E_{c}\left[I_{i}\right] \text { and } \operatorname{Var}_{c}\left[I_{i}\right]\end{array}$ \\
\hline $\begin{array}{r}\text { Empirical inference } \\
\text { simulations sample from the } \\
\text { "reference" distribution }\end{array}$ & Not considered & $\begin{array}{l}\text { Originally in Anselin (1995). } \\
\text { Implemented in Rey \& Anselin (2007). } \\
\text { Used in Bivand and Wong (2018). } \\
\text { Denoted here as } E_{p}\left[I_{i}\right] \text { and } \operatorname{Var}_{p}\left[I_{i}\right]\end{array}$ \\
\hline
\end{tabular}

Fig. 2 Null hypotheses inference methods for local Moran's $I_{i}$ statistic in the literature.

Therefore, we provide a systematic investigation of the relationship between the variance of the $Z\left(I_{i}\right)$ statistics under both randomization assumptions and the local heteroskedasticity in the data. In order to do this, we first revisit classic formulations of the local Moran's $I_{i}$ and present the local heteroskedasticity measure (LOSH), $H_{i}$ that will be used throughout the rest of the paper.

\subsection{Local Moran Statistics}

A local Moran's I statistic, $I_{i}$, can be calculated for each site $i$ using the product of a centered variable $z$ and its spatial lag:

$$
I_{i}=z_{i} \sum_{j} w_{i j} z_{j}
$$

Here, $z_{i}$ is the value of a centered variable $z$ at the "focal" site $i$, and $z_{j}$ is the value of $z$ at a different site, $j$. The spatial relationship between sites $i$ and $j$ is represented by a spatial weight, $w_{i j}$, such that $\sum_{j} w_{i j} z_{j}$ represents a geographically-weighted average of values around site $i$. When the $I_{i}$ statistic is positive, it suggests that $z_{i}$ is on the same side of the mean as its spatial lag, indicating clustering. When negative, it suggests that $z_{i}$ is on opposite sites of the mean from its spatial lag, indicating $i$ is a spatial outlier.

\subsubsection{Local Moran Moments under Total Randomization Null}

Inference depends on the distribution of $I_{i}$. It is on this point where extensive discussion exists. The original analysis Anselin (1995) defines the following moments under (what Sokal et al. (1998) calls) a "total randomization" hypothesis, where all maps of the re-assigned observed data are equally likely. The mean and variance of $I_{i}$ under this null hypothesis are: 


$$
\begin{aligned}
E\left[I_{i}\right] & =\sum_{j \neq i}^{n} \frac{w_{i j}}{n-1} \\
\operatorname{Var}\left[I_{i}\right] & =\frac{w_{i(2)}\left(n-b_{2}\right)}{n-1}+\frac{2 w_{i(k h)}\left(2 b_{2}-n\right)}{(n-1)(n-2)}-\frac{w_{i}^{2}}{(n-1)^{2}}
\end{aligned}
$$

Where $b_{2}$ is the ratio between the fourth and squared second moment, $w_{i(2)}$ is the sum of squared weights (omitting $w_{i i}$ ), and $2 w_{i(k h)}$ is the all-pairs product of weights (omitting self-neighbors):

$$
\begin{aligned}
b_{2} & =\frac{m_{4}}{m_{2}^{2}}=\frac{\sum_{i} z_{i}^{4}}{n} *\left(\frac{n}{\sum_{i} z_{i}^{2}}\right)^{-2} \\
w_{i(2)} & =\sum_{j \neq i} w_{i j}^{2} \\
w_{i(k h)} & =\sum_{k \neq i, h h \neq i, k} \sum_{i k} w_{i h}
\end{aligned}
$$

Note that the expected value and variance of $I_{i}$ vary only according to functions of $w_{i}$. You can tell this is a "total" randomization null because it "depends only on the structure of the connection graph." (Sokal et al., 1998, p. 349)

\subsubsection{Local Moran Moments under Conditional Randomization Null}

However, as Sokal et al. (1998) argues, conditional permutation will also include the value of the process at each site, since we "condition" on $z_{i}$. As argued by Anselin (1995) and implemented in Rey and Anselin (2007), these can obtained directly by simulation, whereby each site has its value of $z_{i}$ fixed, the rest of the map is shuffled, and the spatial lag recomputed. Since this does not admit a simple mathematical expression in terms of $z_{i}$ or $w_{i j}$, we refer to these as $E_{p}\left[I_{i}\right]$ and $\operatorname{Var}_{p}\left[I_{i}\right]$.

Alternatively, Sokal et al. (1998) offers a mathematical statement of these moments. Reprising Equations A7 and A8 from Sokal et al. (1998), the conditional randomization null yields different moments than those in Equation 1; now, they include $z_{i}$ :

$$
\begin{aligned}
E_{c}\left[I_{i}\right] & =-z_{i}^{2} \sum_{j \neq i}^{n} \frac{w_{i j}}{n-1} \\
\operatorname{Var}_{c}\left[I_{i}\right] & =\left[\frac{z_{i}}{m_{2}}\right]^{2}\left[\frac{n}{n-2}\right]\left[w_{i(2)}-\frac{w_{i}^{2}}{n-1}\right]\left[m_{2}-\frac{z_{i}^{2}}{n-1}\right]
\end{aligned}
$$

In this form, we subscript the moments with a $c$ to denote they are obtained with respect to a "conditional randomization" null hypothesis. These means and variances depend both on site values $z_{i}$ and site connectivity $w_{i}$. 


\subsection{Local Moran Z-scores}

Regardless of whether we use the conditional or total randomization null, a local z-score, $Z\left(I_{i}\right)$, is generally calculated. This centers and re-scales each of the $I_{i}$ values to enable comparisons and visualization in aggregate, as in Figure 1. For a null hypothesis, we construct $Z\left(I_{i}\right)$ by removing the mean and scaling by the standard error:

$$
Z\left(I_{i}\right)=\frac{I_{i}-E_{*}\left[I_{i}\right]}{\sqrt{\operatorname{Var}_{*}\left[I_{i}\right]}}
$$

We use the $*$ subscript to indicate that this applies to any of the forms of local moments discussed above.

\subsection{Local Heteroskedasticity}

Local spatial heteroskedasticity (LOSH), $H_{i}$, provides a measure of heterogeneity by focusing on the local structure of dispersion around the mean of a variable $x$. LOSH is calculated as:

$$
H_{i}(d)=\frac{\sum_{j} w_{i j}(d)\left|e_{j}(d)\right|^{2}}{\sum_{j} w_{i j}(d)}
$$

where $\sum_{j} w_{i j}(d)$ is the sum of neighbors within a distance $(d)$ of location $i$. The local residual, $e_{j}$, describes the difference between a site and its surrounding values:

$$
e_{j}(d)=x_{j}-\bar{x}(d), j \in N(i, d)
$$

When $e_{j}$ is large, it indicates that $x_{j}$ is far from its local mean. Thus, when $H_{i}$ is large, it suggests that the Local Sum of Squared Error (LSSE) is large: observations near site $i$ are generally not well described by the mean near $i$, and thus the variance around $i$ is relatively large. Likewise, when $H_{i}$ is small, it means that the LSSE is small, and thus an estimate of variance in the area around $i$ would also be small. Together, this gives an estimate of the local variance, and can be used to characterize areas with higher or lower variance.

\section{Investigating local statistic variance and heteroskedasticity: uncontrolled DGP}

Theoretically speaking, the local variance $H_{i}$ and the variance of local Moran's $I, \operatorname{Var}_{*}\left[I_{i}\right]$, are different; $\operatorname{Var}_{*}$ does not consider the local mean of $z_{i}$, while $H_{i}$ does. But, Bivand and Wong (2018) make a reasonable argument: since conditional randomization methods assume that the variance is constant over space, areas with large local variance may be under-stated by $\operatorname{Var}_{\mathcal{C}}\left[I_{i}\right]$. However, one would also expect to see this in total randomization methods. 


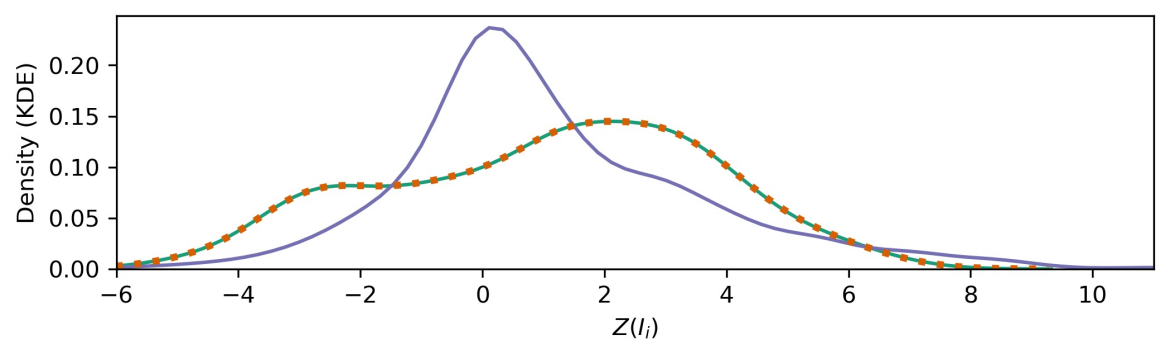

\footnotetext{
_ Empirical "conditional randomization" $Z\left(I_{i}\right)$, corresponds to $\operatorname{Var}_{p}\left[I_{i}\right]$

— Analytical "total randomization" $Z\left(I_{i}\right)$, corresponds to $\operatorname{Var}\left[I_{i}\right]$

" - " Analytical "conditional randomization" $Z\left(l_{i}\right)$, corresponds to $\operatorname{Var}_{c}\left[l_{i}\right]$
}

Fig. 3 Comparison of local Moran's I Z $\left(I_{i}\right)$ scores generated by analytical and conditional permutation methods for unemployment in Camden OAs.

Therefore, to assess the relationship between $\mathrm{LOSH}, H_{i}, \mathrm{Z}\left(I_{i}\right)$, and other component values, we first consider an example with an uncontrolled data generating process. Mirroring Bivand and Wong, we use unemployment data (expressed as a \%) at the Output Area (OA) level provided by Guy Lansley and James Cheshire for a Consumer Daa Research Centre (CDRC) tutorial $^{5}$. We computed local Moran's $I_{i} Z\left(I_{i}\right)$ using the Moran_Local function in the esda submodule (version 2.3.6) of the Python Spatial Analysis Library (PySAL) for the different methods of inference: analytical total randomization $\left(\operatorname{Var}\left[I_{i}\right]\right)$, analytical conditional randomization $\left(\operatorname{Var}_{c}\left[I_{i}\right]\right)$, and empirical conditional permutation $\left(\operatorname{Var}_{p}\left[I_{i}\right]\right)$ (Rey and Anselin, 2007). We used general inverse distance weights for all observations based on polygon centroids and set the number of permutations to 9999 .

We again recreate the motivating figure from Bivand and Wong (2018), with the additional distinction between analytical total randomization and analytical conditional randomization $Z\left(I_{i}\right)$ values (Figure 3 ). This updated figure reveals that analytical conditional randomization and empirical conditional randomization are in near-perfect alignment, as seen in their overplotting in Figure 3. Tabulations of $Z\left(I_{i}\right)$ scores outside -2 to 2 reveal similar strong agreement, with $430(57.4 \%)$ from $\operatorname{Var}_{p}\left[I_{i}\right]$ and $427(57.0 \%)$ from $\operatorname{Var}_{c}\left[I_{i}\right]$. In comparison, the distribution from $\operatorname{Var}\left[I_{i}\right] \mathrm{Z}\left(I_{i}\right)$ appears narrower and identifies fewer scores outside -2 to 2 (267 or $35.6 \%$ ).

We then computed the LOSH, $H_{i}$, for Camden OA unemployment, again using IDW weights. Data visualization and Pearson's correlation coefficient were used to evaluate the strength of the relationship between the different measures of variance for local Moran's $I_{i}$ and $H_{i}$ (Figure 4). There was low to no apparent correlation between variance for the local Moran's $I_{i}$ and $H_{i}$, regardless of which variance of $I_{i}$ was considered $\left(\operatorname{Var}\left[I_{i}\right]\right.$ and $H_{i}: 0.25, \operatorname{Var}_{c}\left[I_{i}\right]$

5 This data is publicly available at https://data.cdrc.ac.uk/tutorial/an-introduction-to-spatial-dataanalysis-and-visualisation-in-r after registering a user account with the CDRC. 

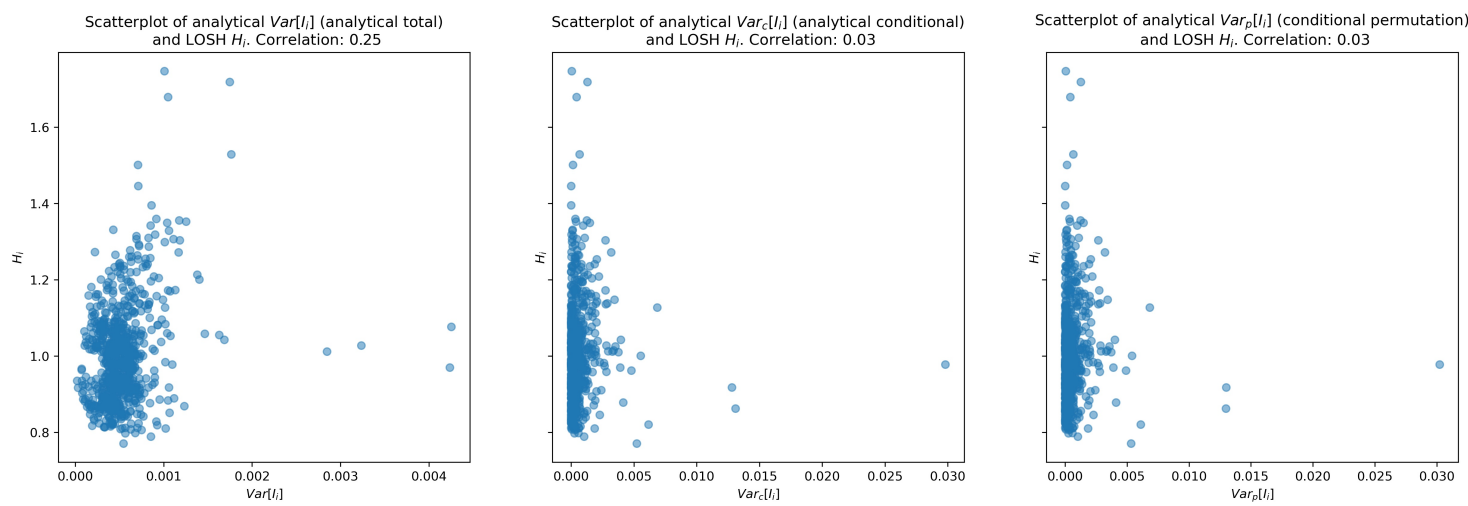

Fig. 4 Scatterplots of different measures of $\operatorname{Var}\left[I_{i}\right]$ and $H_{i}$ for unemployment in Camden OAs.

and $H_{i}: 0.03, \operatorname{Var}_{p}\left[I_{i}\right]$ and $\left.H_{i}: 0.03\right)$. Thus, given the strong correspondence between the analytical and empirical values generated under the same null, it seems likely that the difference in $Z\left(I_{i}\right)$ is due primarily to the implications of the null, not local heteroskedasticity.

\section{Simulated examination of $\operatorname{Var}\left[I_{i}\right]$ and $H_{i}$ : controlled DGP}

To assess this observation more rigorously, it would be useful to examine a process with clear local spatial heteroskedasticity. Therefore, we construct a simulated example with a controlled DGP: a Gaussian random field with induced heteroskedasticity. This Gaussian random field is defined as:

$$
U \sim N_{m}\left(0, K\left(s_{i}, s_{j}\right) * \sigma^{2}\right)
$$

where $m$ is a number of observations, $s_{i}$ is the local site $i, s_{j}$ is the neighboring site $j$, and $K\left(s_{i}, s_{j}\right)$ is an exponential covariance function defined as $K\left(s_{i}, s_{j}\right)=\exp \left(-\frac{\left\|s_{i}-s_{j}\right\|}{h}\right) . h$ is a range parameter, and $\sigma^{2}$ is the variance.

From this Gaussian random field we induce heteroskedasticity by multiplying each random value by its standardized (row/totalrows) $*$ (column/totalcolumns) position. This process allows us to purposefully induce heteroskedasticity in a diagonal pattern across the data. In the top left, the variance is small; in the bottom right, the variance is large. We generate a Gaussian random field of 10000 total observations (e.g., 100 rows and 100 columns) using the GSTools Exponential function (Müller and Schüler, 2019). This process generates both a random field (Figure 5, left) as well as that same random field 

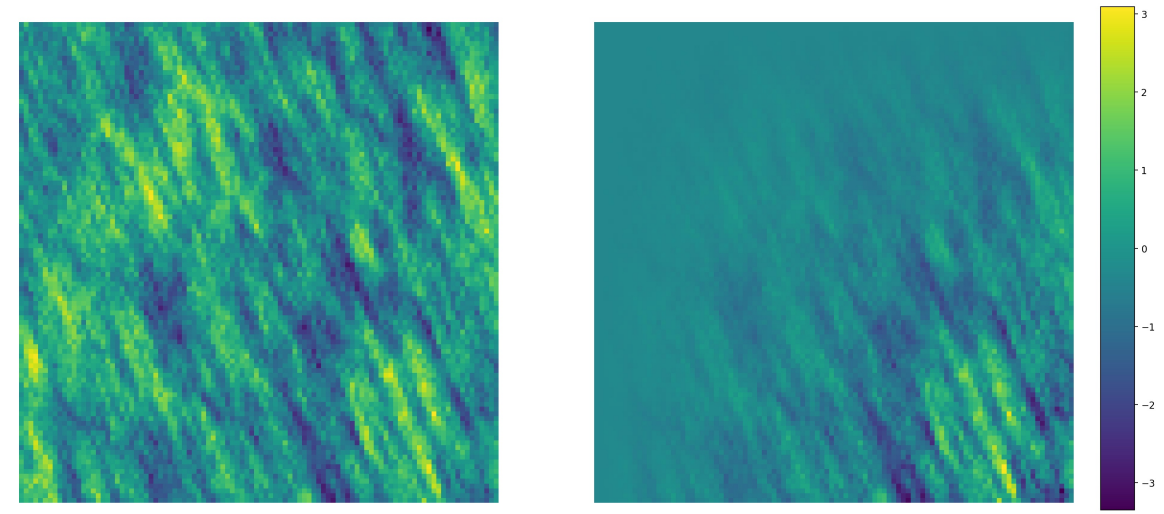

Fig. 5 Randomly generated Gaussian field (left) and that same field with induced heteroskedasticity (right).

with induced heteroskedasticity where the variance increases in magnitude as the row and column position increase (Figure 5, right).

We then repeat the analysis carried out in the the uncontrolled DGP by calculating the analytical total randomization $\left(\operatorname{Var}\left[I_{i}\right]\right)$, analytical conditional randomization $\left(\operatorname{Var}_{c}\left[I_{i}\right]\right)$, and empirical conditional permutation $\left(\operatorname{Var}_{p}\left[I_{i}\right]\right)$ local Moran's $Z\left(I_{i}\right)$ values. As before, we use general IDW weights using the esda Moran Local function, although we reduce the number of permutations to 999 to reduce computational burden. We use the same format from the motivating figure of Bivand and Wong (2018) to show the distribution of $\operatorname{Var}\left[I_{i}\right], \operatorname{Var}_{c}\left[I_{i}\right]$, and $\operatorname{Var}_{p}\left[I_{i}\right] Z\left(I_{i}\right)$ values (Figure 6). For the Gaussian random field with no induced heteroskedasticity (Figure 6, left), the $\operatorname{Var}_{c}\left[I_{i}\right] Z\left(I_{i}\right)$ and $\operatorname{Var}_{p}\left[I_{i}\right] Z\left(I_{i}\right)$ are in strong alignment with respect to both the shape of the distribution and the the number of cells identified as outside -2 to $2\left(\operatorname{Var}_{c}\left[I_{i}\right]\right.$ : 8122 of 10000, and $\operatorname{Var}_{p}\left[I_{i}\right]: 8124$ of 10000, whereas $\operatorname{Var}\left[I_{i}\right]: 6020$ of 10000, ). We observe a similar pattern for the Gaussian random field with induced heteroskedasticity (Figure 6, right), although fewer cells in total are identified as outside -2 to $2\left(\operatorname{Var}_{c}\left[I_{i}\right]: 6134\right.$ of 10000 , and $\operatorname{Var}_{p}\left[I_{i}\right]: 6178$ of 10000, whereas $\operatorname{Var}\left[I_{i}\right]: 3260$ of 10000, ).

Data visualization and Pearson's correlation between measures of variance for the local Moran's $I_{i}$ and LOSH, $H_{i}$, for both Gaussian fields showed some association, although the strength of this association was unclear after only one realization of the random fields (Supplementary Figure 1). The arrangement of values on this one particular Gaussian random field is unlikely to represent the general relationship between the variance components of the local Moran's $I_{i}$ statistic and LOSH $H_{i}$. We need to repeat this analysis over many randomly generated fields to reach a more general understanding of these relationships.

Thus, we generate 10000 Gaussian random fields with induced heteroskedasticity, each time repeating the analytical strategy outlined previously. How- 

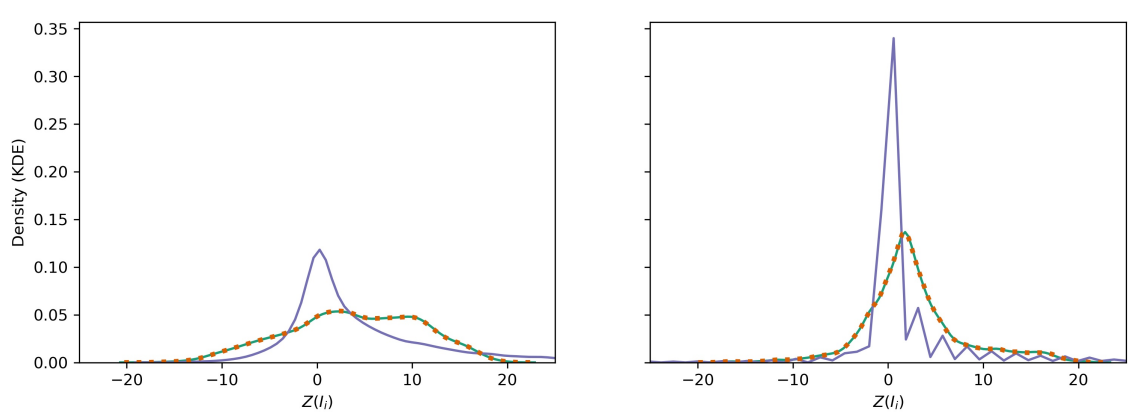

- Empirical "conditional randomization" $Z\left(l_{i}\right)$, corresponds to $\operatorname{Var}_{p}\left[l_{i}\right]$
- Analytical "total randomization" $Z\left(l_{i}\right)$, corresponds to $\operatorname{Var}^{\prime}\left[l_{i}\right]$
. . Analytical "conditional randomization" $Z\left(l_{i}\right)$, corresponds to $\operatorname{Var}_{c}\left[l_{i}\right]$

Fig. 6 Comparison of local Moran's I Z $\left(I_{i}\right)$ scores generated by analytical and conditional permutation methods for methods for a randomly generated Gaussian field (left) and that same field with induced heteroskedasticity (right).

ever, because the IDW weights make use of all neighbors in each calculation and permutation of local Moran's $I_{i}$, repetition of our analysis strategy quickly becomes computationally demanding. Instead of IDW weights we opt for a queen-style spatial weight matrix. The result of the simulations is a distribution of cross-correlations between the variance components of the local Moran's $I_{i}$ statistics and LOSH $H_{i}$. We present the median correlation between components of local Moran's $I_{i}$ and LOSH in Figure 7.

Across all simulations the median correlation between $\operatorname{Var}\left[I_{i}\right]$ and $H_{i}$ was 0.04 (2.5th percentile: $0.01,97.5$ th percentile: 0.08 ). Comparatively, the median correlation between $\operatorname{Var}_{c}\left[I_{i}\right]$ and $H_{i}$ was 0.26 (2.5th percentile: 0.20 ; 97.5th percentile: 0.32 ), and the median correlation between $\operatorname{Var}_{p}\left[I_{i}\right]$ and $H_{i}$ was also 0.26 (2.5th percentile: 0.20 ; 97.5th percentile: 0.32 ) (rounded to the hundredths). The results demonstrated in Figure 6 and Figure 7 suggest that analytical conditional randomization $\left(\operatorname{Var}_{c}\left[I_{i}\right]\right)$ and empirical conditional permutation $\left(\operatorname{Var}_{p}\left[I_{i}\right]\right)$ show strong agreement with each other and a moderately strong association with $\mathrm{LOSH} H_{i}$. In comparison, analytical total randomization $\left(\operatorname{Var}\left[I_{i}\right]\right)$ does not demonstrate a strong association with LOSH $H_{i}$. Thus, in these simulations, too, the difference in results is associated with differences in nulls, not local heteroskedasticity.

\section{Discussion}

This paper set out to investigate an important hypothesis about local statistics made by Bivand and Wong in their recent review of spatial autocorrelation statistics (Bivand and Wong, 2018). The key motivating issue was that there were serious differences in the distribution of $Z\left(I_{i}\right)$ values between analytical and permutation-based methods of inference that may be due to the presence 


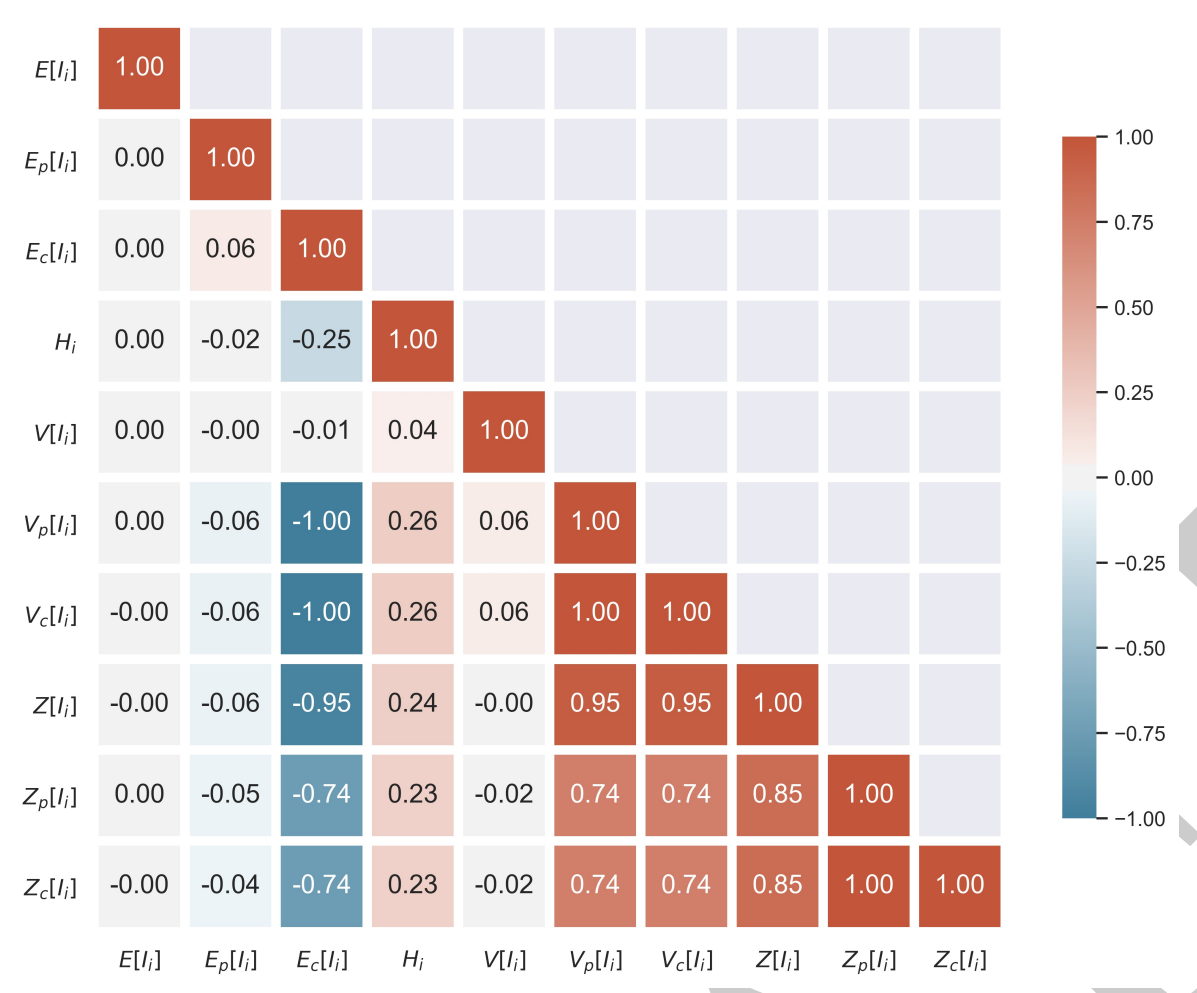

Fig. 7 Median correlation between components of local Moran's $I_{i}$ and LOSH $H_{i}$ calculated over 10,000 Gaussian random fields with induced heteroskedasticity. The local Moran's $I_{i}$ calculations used a queen-style spatial weight matrix and 9999 permutations where applicable. The LOSH $H_{i}$ also used a queen-style spatial weight matrix.

of spatial heteroskedasticity. Namely, areas with larger local variance might be expected to have higher variance in their local statistics.

However, we find that the difference is much more likely to arise as a consequence of different null hypotheses used for local testing, not local heteroskedasticity as such. We see this theoretically through results from Sokal et al. (1998). We verify this empirically in the associations between variance components of the local Moran's $I_{i}$ statistics, such as $\operatorname{Var}\left[I_{i}\right], \operatorname{Var}_{\mathcal{C}}\left[I_{i}\right]$, and $\operatorname{Var}_{p}\left[I_{i}\right]$, and a measure of local spatial heteroskedasticity (LOSH), $H_{i}$. There, we see that the analytical and empirical versions of $Z\left(I_{i}\right)$ built using a common conditional randomization null are highly (or perfectly) correlated, whereas those built from a different null have the differences Bivand and Wong (2018) present.

The present analysis has a few limitations worth discussing. We used the unadjusted values of local Moran's $I_{i}$ as the primary statistic of comparison between analytical and conditional permutation inference, and we did not extensively evaluate how the methods of inference compared when using $\mathrm{p}$-values adjusted via techniques like Bonferroni bounds or the false discov- 
ery rate. Additionally, while we were able to replicate and expand upon the findings of Bivand and Wong, it may be that analytical and conditional permutation inference yield more similar results on datasets not considered in the present analysis.

Bivand and Wong's excellent review identifes several implications of these differences in results that remain pertinent to future users of the local Moran's $I_{i}$ statistic. Principal among these is that the inferential output and subsequent substantive interpretation of results is influenced by the choice of inferential method. Specifically, users might find more clusters using a conditional randomization null than a total randomization null. Future users of the statistic must take care to ensure which inferential method is implemented in their software of choice. This is a separate methodological sensitivity of the statistic that should be considered in conjunction with existing concepts like the false discovery rate or the influence of spatial scale on the analysis (Escamilla et al., 2016). These challenges are not meant to discourage the use of local Moran's $I_{i}$ or a particular inferential method; rather, they should help advance the theoretical justification and methodological choices made by researchers when using local Moran's $I_{i}$ in increasingly popular and public facing (geographic) data science workflows (Singleton and Arribas-Bel, 2019; Wolf et al., 2020).

Future research could investigate whether similar differences in inferential results appear for other statistics of spatial autocorrelation that have the option of several inferential methods (e.g. Lee's $L$ statistic). Certain patterns in the present analysis - such as the tendency for the conditional permutation $Z\left(I_{i}\right)$ distribution to have a longer and wider right tail - remain unexplained. This research contributes additional understanding of the methodological sensitives that surround the area of spatial analysis concerned with "cluster detection", especially when using local Moran's $I_{i}$.

In closing, it is important to stress the theoretical differences. A vast and large body of work has expanded upon Anselin (1995), but the "total" and "conditional" null, in contemporary literature and implementations, are generally distinguished by computation, not conceptualization. In this way, Sokal et al. provides useful tools for us to explain these important differences in the take-away message our estimators offer while presaging contemporary critique about null hypotheses in statistical testing. As a community of practice, spatial analysts should also take note of these critiques.

\section{References}

Anselin, L. (1995). Local Indicators of Spatial Association—LISA. Geographical Analysis, 27(2):93-115.

Anselin, L. and Li, X. (2020). Geographical Analysis, 52(4).

Anselin, L., Syabri, I., and Smirnov, O. (2002). Visualizing Multivariate Spatial Correlation with Dynamically Linked Windows. In New Tools for Spatial Data Analysis: Proceedings of the Specialist Meeting. University of 
California, Santa Barbara: Center for Spatially Integrated Social Science (CSISS).

Assuncao, R. and Reis, E. A. (1999). A new proposal to adjust Moran's I for population density. Statistics in Medicine, 18:2147-2162.

Bivand, R., Anselin, L., Berke, O., Bernat, A., Carvalho, M., Chun, Y., Dormann, C., Dray, S., Halbersma, R., Lewin-Koh, N., et al. (2011). Spdep: Spatial Dependence: Weighting Schemes, Statistics and Models. R package version 0.5-31, URL http:/ /CRAN. R-project. org/package= spdep.

Bivand, R., Müller, W. G., and Reder, M. (2009). Power calculations for global and local Moran's I. Computational Statistics and Data Analysis, 53(8):2859-2872.

Bivand, R. S. and Wong, D. W. (2018). Comparing implementations of global and local indicators of spatial association. Test, 27(3):716-748.

Caldas de Castro, M. and Singer, B. H. (2006). Controlling the false discovery rate: A new application to account for multiple and dependent tests in local statistics of spatial association. Geographical Analysis, 38(2):180208.

Escamilla, V., Hampton, K. H., Gesink, D. C., Serre, M. L., Emch, M., Leone, P. A., Samoff, E., and Miller, W. C. (2016). Influence of detection method and study area scale on syphilis cluster identification in North Carolina. Sexually Transmitted Diseases, 43(4):216-221.

Getis, A. (2008). A history of the concept of spatial autocorrelation: A geographer's perspective. Geographical Analysis, 40(3):297-309.

Lee, S.-I. (2001). Developing a bivariate spatial association measure: An integration of Pearson's r and Moran's I. Journal of Geographical Systems, 3(4):369-385.

Müller, S. and Schüler, L. (2019). GeoStat-Framework/GSTools: Bouncy Blue (Version v1.0.1).

Ord, J. K. and Getis, A. (2001). Testing for local spatial autocorrelation in the presence of global autocorrelation. Journal of Regional Science, 41:441432.

Ord, J. K. and Getis, A. (2012). Local spatial heteroscedasticity (LOSH). Annals of Regional Science, 48(2):529-539.

Rey, S. J. and Anselin, L. (2007). PySAL: A Python library of spatial analytical methods. The Review of Regional Studies, 37(1):5-27.

Singleton, A. and Arribas-Bel, D. (2019). Geographic Data Science. Geographical Analysis, (March):1-15.

Sokal, R., Thompson, B. A., and Oden, N. L. (1998). Geographical Analysis, 30(4):331-354.

Tiefelsdorf, M. (2002). The Saddlepoint Approximation of Moran's I's and Local Moran's Ii's Reference Distributions and Their Numerical Evaluation. Geographical Analysis, 34(3):187-206.

Wasserstein, R. L. and Lazar, N. A. (2016). The ASA's statement on p-values: Context, process, and purpose. The American Statistician, 70(2):129-133.

Wolf, L. J., Fox, S., Harris, R., Johnston, R., Jones, K., Manley, D., Tranos, E., and Wang, W. W. (2020). Quantitative geography III: Future chal- 
lenges and challenging futures. Progress in Human Geography, page 030913252092472.

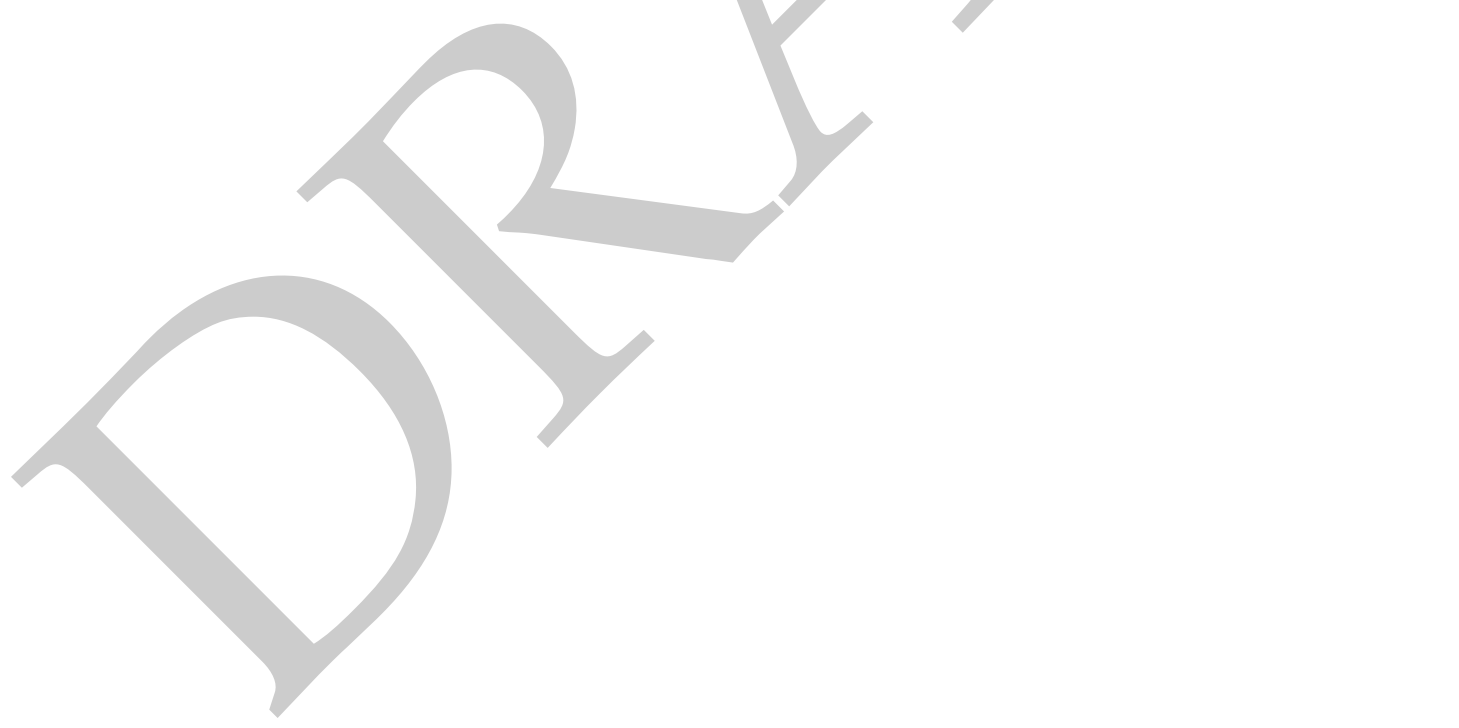

